\section{Case Reports in Ophthalmology}

Case Rep Ophthalmol 2020;11:336-341

DOI: 10.1159/000508391

Published online: July 14, 2020

(C) 2020 The Author(s)

Published by S. Karger AG, Basel

www.karger.com/cop

This article is licensed under the Creative Commons Attribution-NonCommercial 4.0 International License (CC BY-NC) (http://www.karger.com/Services/OpenAccessLicense). Usage and distribution for commercial purposes requires written permission.

\title{
Bilateral XEN Gel Stent Implantation in Juvenile-Onset Open-Angle Glaucoma
}

\author{
Emma Klug David Solá-Del Valle \\ Glaucoma Service, Massachusetts Eye and Ear Infirmary, Boston, MA, USA
}

\section{Keywords}

Juvenile-onset open-angle glaucoma - Minimally invasive glaucoma surgery · XEN Gel Stent . Glaucoma

\begin{abstract}
The purpose of the current case is to report the successful management of juvenile-onset open-angle glaucoma with bilateral XEN Gel Stent (Allegran Inc., Irvine, CA) implantation in a patient first diagnosed during pregnancy with co-existing dysautonomia. Treatment with the XEN Gel Stent provided dramatic reductions in intraocular pressure (IOP), and glaucoma medications sustained up to 23 months postoperatively. The success of this case may suggest that this minimally invasive procedure could be an effective treatment option for younger patients who require substantial reductions in IOP and glaucoma medication burden.
\end{abstract}

(C) 2020 The Author(s)

Published by S. Karger AG, Basel

\section{Introduction}

Juvenile-onset open-angle glaucoma (JOAG) is a rare subset of primary open-angle glaucoma (POAG) with an age at onset between 5 and 35 years [1]. It is often associated with a rapidly progressive disease that can be refractory to medical therapy. Surgical intervention is usually required to control intraocular pressure (IOP) and slow disease progression.

KARGER
David Solá-Del Valle

Glaucoma Service, Massachusetts Eye and Ear Infirmary

243 Charles Street

Boston, MA 02114 (USA)

David_Sola-DelValle@meei.harvard.edu 


\section{Case Reports in Ophthalmology}

Minimally invasive, angle-based procedures are typically preferred in JOAG cases as they restore trabecular meshwork function without bypassing it via the creation of a filtering bleb [2]. They also avoid manipulation of the conjunctiva that could be needed for future surgical intervention. However, these procedures are generally less effective in reducing IOP compared to trabeculectomy and tube-shunt procedures [3].

The purpose of the current case report is to introduce another minimally invasive glaucoma surgery, the ab interno XEN Gel Stent, for the treatment of JOAG. While it requires bleb formation, an ab interno approach can provide excellent IOP reduction while sparing most of the patient's conjunctiva. To the best of our knowledge, there is only one existing report of the use of XEN Gel Stent in JOAG, with a focus on its management during pregnancy. The followup period of this report was limited to 2 months [4]. Herein, we report the 23-month followup of a non-pregnant JOAG patient who underwent successful XEN Gel Stent implantation in both eyes.

\section{Case Presentation}

A 48-year-old Caucasian woman presented to the Glaucoma Service at the Massachusetts Eye and Ear Infirmary in August 2018 for a routine 6-month IOP check for bilateral, mild-stage JOAG. The patient denied any pain or changes in vision. Her best-corrected visual acuity was 20/20 OD and 20/20 OS. IOP measurements were $19 \mathrm{~mm} \mathrm{Hg}$ in the right eye and $38 \mathrm{~mm} \mathrm{Hg}$ in the left eye. Gonioscopy showed grade 3 open angles. The anterior chamber and the lens were clear bilaterally, and the cup-to-disc ratios were $0.75 \mathrm{OD}$ and $0.70 \mathrm{OS}$. Humphrey visual field analysis revealed a full field OD and scattered, non-specific defects OS. Optical coherence tomography showed an average retinal nerve fiber layer thickness of $65 \mu \mathrm{m}$ OD and $61 \mu \mathrm{m}$ OS.

The patient's glaucoma had been treated at an outside institution where elevated IOP was first noted at the age of 30 during her first pregnancy. Her maximum recorded IOP was $25 \mathrm{~mm}$ $\mathrm{Hg}$ OD and $47 \mathrm{~mm} \mathrm{Hg}$ OS. She had previously undergone selective laser trabeculoplasty OU in 2007, and again OS in 2017. Her glaucoma was currently being treated with 4 topical medications OU: latanoprost q.h.s., timolol $0.5 \%$ b.i.d., brinzolamide t.i.d., and brimonidine t.i.d. This reliable patient endorsed excellent adherence to this complex medication regimen.

The patient had a positive family history of glaucoma, and she had received long-term oral prednisone for possible systemic lupus erythematosus in 2012, 6 years prior to presentation. She had not received any systemic steroids since that time. Genetic testing to rule out myocilin-associated JOAG was not performed. It is therefore possible that her glaucoma was attributable to multiple mechanisms, including both JOAG and steroid-induced glaucoma. Notably, the patient also had a current diagnosis of dysautonomia, a disorder of the autonomic nervous system that usually involves failure of the sympathetic or parasympathetic components of the autonomic nervous system [5]. She took many measures to manage a host of symptoms associated with the disorder, including variable heart rates and blood pressures. Her condition was being treated with pyridostigmine bromide, an orally active cholinesterase inhibitor.

When the patient's IOP was markedly elevated in August 2018, latanoprost was switched to latanoprostene bunod q.h.s. OU, netarsudil was added q.h.s. OU, and she was tentatively scheduled for a trabeculectomy with mitomycin-C OS. With these changes in the medication regimen, her IOP decreased to $17 \mathrm{~mm} \mathrm{Hg}$ OD and $29 \mathrm{~mm} \mathrm{Hg}$ OS prior to surgery. The trabeculectomy was cancelled in favor of a minimally invasive procedure according to patient preference and more in line with her age and glaucoma stage. 


\section{Case Reports in Ophthalmology}

Case Rep Ophthalmol 2020;11:336-341 DOI: $10.1159 / 000508391$

C 2020 The Author(s). Published by S. Karger AG, Basel www.karger.com/cop

Klug and Solá-Del Valle: XEN Gel Stent Implantation in JOAG

After consideration of the risks and benefits of various approaches, the surgeon and patient decided to proceed with XEN Gel Stent implantation OS in September 2018, in an ab interno fashion without opening the conjunctiva. Approximately $0.1 \mathrm{~mL}$ of mitomycin 0.4 $\mathrm{mg} / \mathrm{mL}$ were injected subconjunctivally. On postoperative day 1, IOP measurements were 15 $\mathrm{mm} \mathrm{Hg}$ OD and $02 \mathrm{~mm} \mathrm{Hg} \mathrm{OS}$. The anterior chamber was deep in both eyes. The postoperative course consisted of 1 week of moxifloxacin therapy and atropine while the eye was hypotonous (IOP $<5 \mathrm{~mm} \mathrm{Hg}$ ). Prednisolone acetate $1 \%$ was given 8,6 , and 4 times a day for 2 weeks each and was then tapered over the next 4 weeks. The patient remained on prednisolone acetate $1 \%$ once a day for an additional 2 weeks. Ketorolac was also tapered over 4 weeks.

While the left eye healed from surgery, IOP was maintained in the mid-teens in the right eye on the previously described topical regimen. In December 2018, the surgeon and the patient decided to proceed with XEN Gel Stent implantation in the right eye in an effort to decrease her glaucoma medication burden. The procedure was performed in a similar manner to above. On postoperative day 1, IOP measurements were $05 \mathrm{~mm} \mathrm{Hg}$ OD and $11 \mathrm{~mm} \mathrm{Hg}$ OS. The stent was slightly tented up in the conjunctiva, but it was successfully repositioned with a Q-tip. The postoperative course was similar to that of the left eye with the exception of ketorolac. After the initial tenting of the stent, ketorolac was avoided for fear of stent exposure, as significantly greater rates of conjunctival contraction have been observed in patients who received postoperative ketorolac compared to those who received steroid treatment after tube-shunt surgery [6].

At postoperative week 7.5, the patient presented to the clinic with an IOP of $19 \mathrm{~mm} \mathrm{Hg}$ in the right eye off all glaucoma medications. Needling with subconjunctival injection of 5-fluorouracil (5-FU) was performed OD to reduce IOP and prevent scar tissue formation. The patient's IOP dropped to $14 \mathrm{~mm} \mathrm{Hg}$ following the procedure. Over the next 16 months, IOP ranged from $05-19 \mathrm{~mm} \mathrm{Hg}$ OD and $02-15 \mathrm{~mm} \mathrm{Hg}$ OS off all glaucoma medications. Her bestcorrected visual acuity remained 20/20 in each eye, and her visual fields remained stable and full in both eyes with scattered, non-specific defects OS. On optical coherence tomography, her average retinal nerve fiber layer thickness ranged from 58 to $64 \mathrm{OD}$ and from 58 to $63 \mathrm{OS}$. At the last follow-up in July 2020, her IOP measurements were $13 \mathrm{~mm} \mathrm{Hg}$ in each eye off all glaucoma medications. Images of the patient's filtering bleb in the right eye as of January 2020 are shown in Figure 1. The left eye had a similar appearance to the right.

\section{Discussion}

This case illustrates the successful management of JOAG with XEN Gel Stent implants. Both eyes achieved stable IOP control off all glaucoma medications up to 23 months postoperatively. At the most recent follow-up visit, IOP was reduced by $4 \mathrm{~mm} \mathrm{Hg} \mathrm{(24 \% )} \mathrm{OD} \mathrm{and} 16$ $\mathrm{mm} \mathrm{Hg} \mathrm{(55 \% )} \mathrm{OS} \mathrm{from} \mathrm{preoperative} \mathrm{measurements,} \mathrm{and} \mathrm{the} \mathrm{number} \mathrm{of} \mathrm{IOP-lowering} \mathrm{medi-}$ cations was reduced by 5 in each eye.

As mentioned previously, angle-based procedures such as goniotomy, ab interno trabeculectomy, or trabeculotomy are typically deployed as first-line treatment for JOAG. Specifically, Arora et al. [7] observed mean reductions of $10 \mathrm{~mm} \mathrm{Hg}$ in IOP and 0.7 medications 12 months after ab interno trabeculectomy with the trabectome (NeoMedix Corp., Tustin, CA). Moreover, Yeung and Walton [2] observed a comparative $12.7 \mathrm{~mm} \mathrm{Hg}$ reduction in IOP after goniotomy. IOP has also been shown to be reduced by 12.5-16 mm Hg following gonioscopyassisted transluminal trabeculotomy, with reductions in medications ranging from 1.3-1.74 $[8,9]$. More recently, Khouri et al. [10] suggested ab interno trabeculectomy with the Kahook 
dual blade as an alternative to goniotomy, after achieving a $>30 \%$ reduction in IOP 18 months postoperatively in a case report of a 14-year-old male with JOAG.

The substantial reductions in IOP and glaucoma medications observed in the current case align more closely with those reported following ab externo trabeculectomy in JOAG patients, which range from 16-22 mm Hg and 2.3-2.8 medications [11-13]. However, ab externo trabeculectomy is not recommended as a first-line treatment for JOAG despite the potential for greater IOP reduction $[2,7]$. Rather, angle-based procedures are preferred as they spare the conjunctiva and avoid the potential of life-long bleb-related complications $[2,7,8]$. As bleb morphology is critical to ocular health, the potential for long-term bleb-related complications should be emphasized when discussing surgical options with these young patients.

The older age of the JOAG patient described in the current case (48 years) provided a stronger basis for the use of a procedure that creates a filtering bleb and could also provide potentially greater reductions in IOP and medication use. With that said, Smith et al. [14] reported the successful management of congenital glaucoma in 3 young children, with stable control of IOP off all medications and favorable bleb morphology up to 2 years after XEN surgery.

To the best of our knowledge, there is only one existing report of the use of bilateral XEN Gel Stents in JOAG. Similar to the current case, the authors report substantial reductions in IOP from $>40 \mathrm{~mm} \mathrm{Hg}$ preoperatively to $14 \mathrm{~mm} \mathrm{Hg}$ in both eyes 2 months postoperatively [4]. Unlike the current case, their procedure was performed without intraoperative mitomycin-C due to pregnancy. The patient subsequently required multiple 5-FU augmented needling procedures. In contrast, our JOAG patient required a single needling procedure with subconjunctival 5-FU in the right eye.

The need for both postoperative needling and 5-FU injections following XEN Gel Stent insertion is fairly well documented [15]. Specifically, in several prospective studies of XEN insertion with subconjunctival mitomycin-C needling rates have ranged from 30.7-43.2\%, although it has been reported as low as $2.4 \%$ [16-21]. In the present case, needling with an injection of 5-FU successfully reduced the IOP in the right eye from 19 to $14 \mathrm{~mm} \mathrm{Hg}$. Evidence suggests that 5-FU augmented needling revisions are both a safe and effective option for bleb management, with the potential for substantial IOP reductions [22].

\section{Conclusion}

The current case introduces the XEN Gel Stent as a minimally invasive treatment option for JOAG patients. Bilateral XEN Gel Stent implantation reduced this patient's IOP by $24 \%$ OD and $55 \%$ OS, and reduced the patient's glaucoma medication burden by $100 \%$ OU. The XEN Gel Stent may potentially provide greater reductions in IOP and glaucoma medication burden compared to angle-based procedures that are currently recommended as first-line treatment for JOAG. The XEN Gel Stent may particularly benefit JOAG patients who - while still young in comparison to the average POAG patient - may be of an age at which topical and systemic therapy has proved insufficient, and substantial reductions in IOP and medication burden are needed. 


\section{Case Reports in Ophthalmology}

\begin{tabular}{l|l}
\hline DOI: $10.1159 / 000508391$ & (c) 2020 The Author(s). Published by S. Karger AG, Basel
\end{tabular}
www.karger.com/cop

Klug and Solá-Del Valle: XEN Gel Stent Implantation in JOAG

\section{Acknowledgements}

The authors are grateful to Mari Chachanidze and Abraham Nirappel for their continued support and dedication to adjacent projects.

\section{Statement of Ethics}

The patient consented to the publication of this case and the images therein in writing. All research was conducted in accordance with the Declaration of Helsinki.

\section{Disclosure Statement}

The authors declare that they have no conflicts of interest to disclose.

\section{Funding Sources}

This research was generously funded by Stephen Traynor and Charles and Anne Gifford.

\section{Author Contributions}

D.S.-V. was the attending physician of the case. E.K. researched the case, and both authors contributed to the writing and revising process.

\section{References}

1 Turalba AV, Chen TC. Clinical and genetic characteristics of primary juvenile-onset open-angle glaucoma (JOAG). Semin Ophthalmol. 2008 Jan-Feb;23(1):19-25.

2 Yeung HH, Walton DS. Goniotomy for juvenile open-angle glaucoma. J Glaucoma. 2010 Jan;19(1):1-4.

3 Richter GM, Coleman AL. Minimally invasive glaucoma surgery: current status and future prospects. Clin Ophthalmol. 2016 Jan;10:189-206.

4 Zehavi-Dorin T, Heinecke E, Nadkarni S, Green C, Chen C, Kong YX. Bilateral consecutive Xen gel stent surgery during pregnancy for uncontrolled early-onset primary open angle glaucoma. Am J Ophthalmol Case Rep. 2019 Jul;15:100510.

5 Dysautonomia Symptoms. Treatment \& More | Cleveland Clinic. Available from: https://my.clevelandclinic.org/health/articles/6004-dysautonomia. Accessed November 15, 2019.

6 Yuen D, Buys Y, Jin YP, Alasbali T, Smith M, Trope GE. Corticosteroids versus NSAIDs on intraocular pressure and the hypertensive phase after Ahmed glaucoma valve surgery. J Glaucoma. 2011 Sep;20(7):439-44.

7 Arora S, Maeda M, Francis B, Maeda M, Sit AJ, Mosaed S, et al. Efficacy and safety of ab interno trabeculectomy in juvenile open-angle glaucoma. Can J Ophthalmol. 2018 Oct;53(5):482-6.

8 Grover DS, Smith O, Fellman RL, Godfrey DG, Butler MR, Montes de Oca I, et al. Gonioscopy assisted transluminal trabeculotomy: an ab interno circumferential trabeculotomy for the treatment of primary congenital glaucoma and juvenile open angle glaucoma. Br J Ophthalmol. 2015 Aug;99(8):1092-6.

9 Dao JB, Sarkisian SR Jr, Freedman SF. Illuminated microcatheter-facilitated 360-degree trabeculotomy for refractory aphakic and juvenile open-angle glaucoma. J Glaucoma. 2014 Sep;23(7):449-54.

10 Khouri AS, Zhu Y, Sadek H. Ab interno trabeculectomy with the dual blade in juvenile open-angle glaucoma. Eur J Ophthalmol. 2019 Dec;1120672119892440.

11 Tsai JC, Chang HW, Kao CN, Lai IC, Teng MC. Trabeculectomy with mitomycin C versus trabeculectomy alone for juvenile primary open-angle glaucoma. Ophthalmologica. 2003 Jan-Feb;217(1):24-30. 


\section{Case Reports in Ophthalmology}

12 Oh EK, Lee EJ, Jeoung JW, Kim SH, Kim TW, Park KH, et al. Long-Term Outcomes of Trabeculectomy in Korean Patients with Juvenile Open-Angle Glaucoma. J Korean Ophthalmol Soc. 2014;55(2):252.

13 Pathania D, Senthil S, Rao HL, Mandal AK, Garudadari CS. Outcomes of trabeculectomy in juvenile open angle glaucoma. Indian J Ophthalmol. 2014 Feb;62(2):224-8.

14 Smith OU, Grover DS, Emanuel ME, Godfrey DG, Fellman RL. XEN Gel Stent in Pediatric Glaucoma. J Glaucoma. 2020 Apr;29(4):e19-22.

15 Midha N, Rao HL, Mermoud A, Mansouri K. Identifying the predictors of needling after XEN gel implant. Eye (Lond). 2019 Mar;33(3):353-7.

16 De Gregorio A, Pedrotti E, Russo L, Morselli S. Minimally invasive combined glaucoma and cataract surgery: clinical results of the smallest ab interno gel stent. Int Ophthalmol. 2018 Jun;38(3):1129-34

17 Galal A, Bilgic A, Eltanamly R, Osman A. XEN Glaucoma Implant with Mitomycin C 1-Year Follow-Up: result and Complications. J Ophthalmol. 2017;2017:5457246.

18 Pérez-Torregrosa VT, Olate-Pérez Á, Cerdà-Ibáñez M, Gargallo-Benedicto A, Osorio-Alayo V, Barreiro-Rego A, et al. Combined phacoemulsification and XEN45 surgery from a temporal approach and 2 incisions. Arch Soc Esp Oftalmol. 2016 Sep;91(9):415-21.

19 Schlenker MB, Gulamhusein H, Conrad-Hengerer I, Somers A, Lenzhofer M, Stalmans I, et al. Efficacy, Safety, and Risk Factors for Failure of Standalone Ab Interno Gelatin Microstent Implantation versus Standalone Trabeculectomy. Ophthalmology. 2017 Nov;124(11):1579-88.

20 Sheybani A, Lenzhofer M, Hohensinn M, Reitsamer H, Ahmed II. Phacoemulsification combined with a new ab interno gel stent to treat open-angle glaucoma: pilot study. J Cataract Refract Surg. 2015 Sep;41(9):19059.

21 Sheybani A, Dick HB, Ahmed II. Early Clinical Results of a Novel Ab Interno Gel Stent for the Surgical Treatment of Open-Angle Glaucoma. J Glaucoma. 2016 Jul;25(7):e691-6.

22 Wałek E, Przeździecka-Dołyk J, Helemejko I, Misiuk-Hojło M. Efficacy of postoperative management with 5fluorouracil injections after XEN Gel Stent implantation. Int Ophthalmol. 2020 Jan;40(1):235-46.
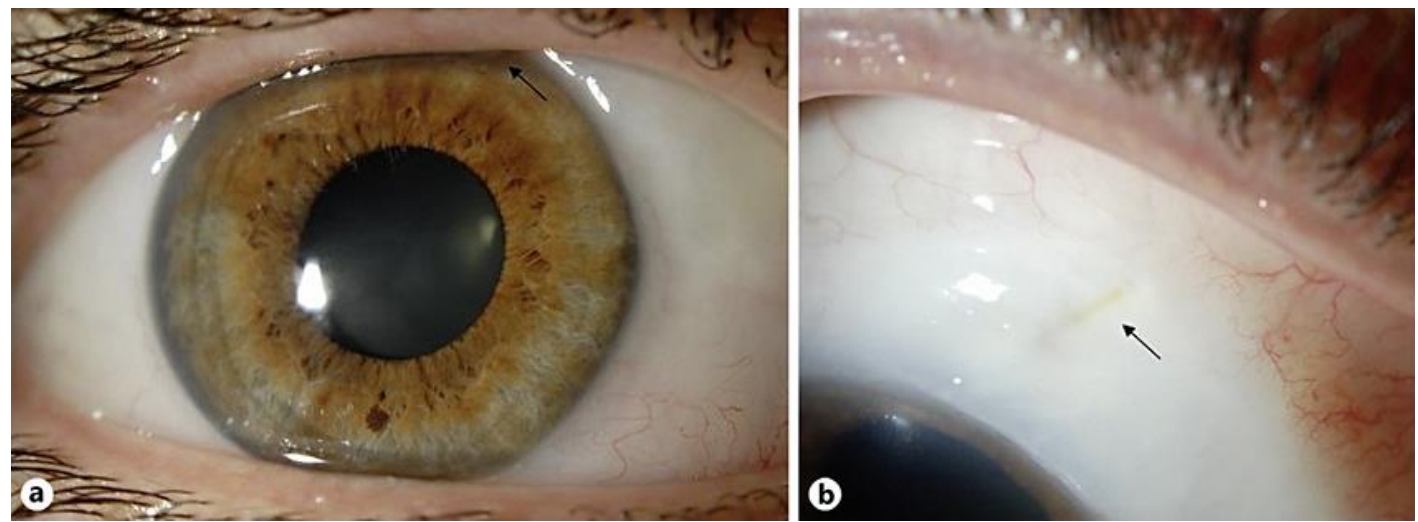

Fig. 1. Images of the filtering bleb in the right eye 16 months postoperatively. Arrows indicate the visible XEN Gel Stent. a View of the right eye head-on. b View of the right eye with the patient looking down. 